\title{
Changing standards for drug approval: A longitudinal analysis of conditional marketing authorisation in the European Union
}

\author{
Jarno Hoekman*, Wouter Boon \\ Innovation Studies Group, Copernicus Institute of Sustainable Development, Faculty of Geosciences, Utrecht University, the Netherlands
}

\section{A R T I C L E I N F O}

\section{Keywords:}

Pharmaceuticals

Regulation

Drug approval

Standards

European Union

\begin{abstract}
A B S T R A C T
Drug regulatory agencies around the world increasingly implement expedited regulatory pathways allowing for approval of medicines that intend to address unmet medical needs based on lower evidentiary standards than would be conventionally required. Few studies have investigated how companies and regulators utilise these pathways. We therefore conducted a longitudinal analysis of the emergence and implementation of the conditional marketing authorisation (CMA) instrument in the European Union. Drawing on archival documents, procedural data and interviews, we show that there was substantial ambiguity among regulators and companies about how to strike a new balance between evidentiary requirements and patient needs. As ambiguities were left unresolved, parties became reluctant to use CMA and in the majority of procedures did not use the pathway in a prospectively planned fashion. Rather, CMA became an option for regulators and companies to apply when submitted data were not strong enough to justify standard approval. Particularly, incumbent companies profited from this. The results stress the challenges of realising institutional change in drug regulation by showing how interest-driven actors can act upon ambiguities in attempts to shape regulatory outcomes and stretch rule interpretations.
\end{abstract}

\section{Introduction}

This paper examines processes of change in drug regulation that challenge conventional standards for drug approval. Worldwide, drug regulatory agencies have implemented a number of expedited regulatory pathways that provide pharmaceutical companies with flexible options for clinical development, particularly for drugs that are intended to address unmet medical needs (Baird et al., 2014). Expedited regulatory pathways stimulate commitments between companies and regulatory agencies during clinical development, shift parts of evidence generation on drugs' benefits and risks from the pre-to the post-authorisation phase, and allow for drug approval based on lower evidentiary standards than conventionally required (Liberti, 2017). The use of these pathways has expanded considerably over the years. Since 2014, the majority of new molecular entities approved by the Food and Drug Administration (FDA) is authorised via an expedited pathway (GAO, 2015), and the recent enactment of instruments facilitating drug development in the European Union (EU) and Japan suggest that other agencies are mimicking this experience (Kondo et al., 2017). In doing so, regulators are gradually extending their role from being mere gatekeepers that safeguard public health to being enablers of drug development (Ehmann et al., 2013).

The key challenge for authorities when implementing expedited pathways is the act of balancing demands for timely access to drugs and the generation of sufficient evidence on their benefits and risks (Eichler et al., 2008). In striking this balance, regulators are often accused of having "just two speeds of approval - too fast and too slow" (Hamburg and Sharfstein, 2009, p. 2494). On the one hand, regulators have been criticised for being overly risk-averse by withholding access to potentially life-saving drugs, e.g. during the early HIV/AIDS crisis (Daemmrich, 2004) or the approval of drugs for small patient populations (de Vrueh et al., 2013). On the other hand, critics have argued that the recent expansion of expedited pathways facilitates the approval of drugs that show only modest evidence of clinical efficacy, a higher likelihood of serious safety issues that remain undetected upon approval, and uncertainty regarding added therapeutic value and the extent to which drugs address unmet medical needs (Davis and Abraham, 2013; Kesselheim et al., 2015; Mostaghim et al., 2017). Concerns are exacerbated by the complexity of generating evidence on the clinical effects of drugs once they are approved on the market. Postmarketing commitments requested by regulators are often not finished in time and do not always deliver what was expected (Hoekman et al.,

\footnotetext{
* Corresponding author. Innovation Studies Group, Copernicus Institute of Sustainable Development, Faculty of Geosciences, Utrecht University, Heidelberglaan 2, P.O. Box 80.115 Utrecht, the Netherlands.

E-mail address: j.hoekman@uu.nl (J. Hoekman).
} 
2016; Naci et al., 2017). Moreover, exit strategies for drugs that turn out to be unsafe or ineffective are not always in place (Braillon and Menkes, 2016).

What complicates these challenges are different viewpoints on the acceptance of uncertainties about the therapeutic value of drugs and ambiguities about appropriate standards for drug approval. We know however little about how regulators and companies deal with these uncertainties and ambiguities in attempts to strike a new balance between patient needs and evidence generation. This knowledge gap is particularly pertinent for EU drug development and regulation. Prior work has examined the macro-political drivers of regulatory change in the EU leading up to the establishment of the European Medicines Agency (EMA) and the centralised procedure in 1995 against the background of a move towards a single European market (Abraham and Lewis, 2000). Recent work also compared drivers of expedited pathway emergence in the EU and United States (US) and illustrated how these pathways have been implemented in the US based on case-studies of Iressa and Lotronex (Davis and Abraham, 2013). We contribute to this work by tracing the implementation of the conditional marketing authorisation (CMA) pathway in the EU as a prominent example of a European expedited pathway. The study's analytical focal point are ambiguities of standards for expedited approval experienced by regulators and companies during implementation. We document how these ambiguities provided scope for strategic and interest-driven discretionary action by both parties, and how this in turn affected regulatory outcomes and stretched rule interpretations.

\section{Contribution}

This study contributes to a body of literature examining how organisations, interests and institutional conditions drive regulatory standard-setting on expedited drug approval (Carpenter, 2004a; Daemmrich, 2004; Davis and Abraham, 2013). Following Abraham (1995), change in drug approval standards can be examined through macro-political studies of the formulation and adoption of legal standards by the legislative (European Parliament and Council in the EU) and executive (European Commission in the EU) branches of government as well as micro-sociological studies of the implementation and interpretation of these standards by the bureaucratic branch (EMA in the EU). We focus on the latter and examine how ambiguity of standards triggered strategic actions of and commitments between regulators and companies during the implementation of CMA in the EU.

In most jurisdictions including the $\mathrm{EU}$, drug regulatory agencies enjoy substantial discretionary autonomy from their political principals to implement legal standards. This autonomy follows from a choice to separate concerns of scientific assessment from political priority-setting (Irwin et al., 1997) and the granting of de-facto power to regulatory agencies to issue soft law instruments such as scientific guidance and communications with regulatees and other parties (Chiti, 2013). Discretionary autonomy provides agencies with the necessary flexibility to deal with the inherent complexities and uncertainties of science-based decision-making on a case-by-case basis. It however also opens up the possibility for ambiguity in the implementation process defined as variability in interpretations and normativities of what legal standards exactly afford and how to make them operational in scientific assessment procedures.

Earlier studies on expedited pathways in the US have shown how approval standards can be ambiguous with regard to the type of technoscientific evidence that is permitted as a basis for market approval and the extent to which drugs need to offer significant therapeutic advance over existing treatment (Davis and Abraham, 2013). These studies have documented how the emergence of expedited approval pathways in the US has been associated with acceptance of techno-scientific evidence of lower standards, and weak accounting systems to demonstrate therapeutic advance of new molecular entities (see also Kesselheim et al., 2015). Based on a study of the re-introduction of Lotronex on the US market, Davis and Abraham (2013) argue that these developments are driven by increased flexibility in approval options for companies, pressure on authorities from political principals and industry, the latter being the 'client' for rapid approval of drugs, and the growing capacity of patient groups to challenge evidence, sometimes collaboratively with companies.

This study documents ambiguities of expedited approval standards in the EU, specifically focusing on how these ambiguities emerged, were acted upon by regulators and companies, and shaped regulatory outcomes and rule interpretations. Theories of regulatory change provide two perspectives on how ambiguity will evolve during implementation of regulation. On the one hand, ambiguity might be a temporary feature that can be resolved by setting precedents, clarifying rules through guidance and communications, and processes of social learning (Grin and Loeber, 2006). As regulators, companies and other parties engage in dialogue during implementation, they will develop shared understandings of regulatory concepts, values, priorities and associated roles and responsibilities. In the EU, this perspective resonates with adaptive approaches to drug regulation (Eichler et al., 2012) that rely on "the participation of multiple stakeholders [...] and creation of a more systematic system of dialogue and exchange of underlying principles during development that are applied by each of the participants" (Ehmann et al., 2013, p. 426).

On the other hand, ambiguity of standards might be a more permanent and difficult to resolve feature as actors will act upon ambiguities according to their own interests (Mahoney and Thelen, 2010). Abraham $(1995,2008)$ distinguishes between the interests of patients and the wider public in approval of drugs with an optimal benefit-risk ratio, and the interests of firms, though not always overriding, in maximising profits. Carpenter (2004a) adds that regulators and bureaucratic leaders also have strategic interests to safeguard their reputation by balancing efficient approval with the prevention of safety scandals. Thus, permanent ambiguity is triggered by the distributional consequences of regulation and is visible in attempts by companies to capture authorities, and by authorities to insulate themselves from interest groups (Ossege, 2015) or co-opt them (Carpenter, 2004b).

Importantly, firms' interests are not monolithic, as larger or more established pharmaceutical companies differ from smaller and newer ones by e.g. greater revenues, diversified product portfolios and enhanced financial risk tolerance (Carpenter, 2004b). Incumbent firms might experience less ambiguity about standards as they have already invested considerably in regulatory capabilities and relationships. Following private interest capture theory (Stigler, 1971), these incumbents are often served by maintenance of the status-quo and the promotion of standards that are difficult to meet for smaller less resourceful companies. At the same time, advantages for large firms might exist when companies have not 'captured' an agency, simply because regulators see the experience of companies as a signal of unobserved product quality or because regulators protect early entrants through political co-optation by organised consumer groups (Carpenter, 2004b). Thus, systematic advantages for incumbent parties could point to private interest capture as well as protection of incumbent parties without capture.

In the following we examine how ambiguity played a role during the design and implementation of CMA and how regulators and companies dealt with this ambiguity.

\section{Methodology}

We conducted a longitudinal case-study of the CMA regulation focusing on the formulation and implementation of approval standards and building on earlier work that we conducted on this instrument (Boon et al., 2010; Hoekman et al., 2015). We cover a full regulatory cycle consisting of problem definition, agenda-setting, formulation of regulation, implementation and evaluation. Initiation of the first evaluation of the centralised marketing authorisation (MA) procedure at EMA in 2001 marks the start of the problem definition phase. 
Evaluation of the CMA regulation resulting in changes to the CMA guideline in 2016 marks the end of the evaluation phase.

\subsection{Data sources}

We gathered data from primary and secondary sources covering regulation-level events and individual drug assessment procedures. First, archival documents were collected through internet searches and access to document requests at the European Commission (EC). Sources included, amongst others, draft and final versions of European regulations and EMA guidelines; impact assessments; stakeholder responses to public consultations and minutes of the Committee for Medicinal Products for Human Use (CHMP) which is the expert-body at EMA responsible for assessing drug applications.

Second, we constructed a dataset containing procedural characteristics of MA applications for all innovative drugs and all orphan medicinal products covering the period April 2006 (first CMA use) to August 2016. The dataset contains 376 applications including all applications that were granted conditional or standard MA, and those that were refused by the CHMP or withdrawn by the applicant before a final opinion of CHMP was reached. Given the specificity of data requirements, we excluded vaccines and drugs involving a request and/or granting of authorisation under exceptional circumstances, the latter being a pathway used for drugs for which the collection of comprehensive evidence is deemed impossible and/or unethical. European Public Assessment Reports and EMA Annual Reports were searched to collect data on product characteristics, approval decisions, review timelines, CHMP voting behaviour, and whether companies discussed with regulators in scientific advice meetings prior to assessment procedures.

Third, 16 interviews with regulators, policy officials and senior staff of pharmaceutical companies on the use of CMA serve as background material. Interviews were conducted in two rounds during 2010 and 2014/2015 initially to support findings published in Boon et al. (2010) and Hoekman et al. (2015). Interviewees were selected based on their direct involvement in designing, implementing and utilizing CMA taking into account variation in organisations and roles. Interviews aimed to reconstruct key decisions made by interviewees in the design and utilization of CMA and lasted for about $60 \mathrm{~min}$. Oral consent was sought before interviews were started, the interviews were subsequently audiotaped, fully transcribed and checked by the respondents for mistakes.

\subsection{Analysis}

Our analytical focal points are ambiguities in the eligibility and approval criteria for granting CMA. We examined how these ambiguities 1) emerged during the law-making process, 2) were acted upon in drug assessment procedures by regulators and companies, and 3) affected regulatory outcomes and triggered further regulatory change in attempts to resolve them. Fig. 1 visualises the eligibility and approval criteria for granting CMA and compares the design of CMA with standard MA in the EU.

First, we reconstructed key events over the regulatory cycle (Section 4.1) and examined how ambiguity of standards emerged in this process (Section 4.2). Both authors comprehensively read all archival documents and jointly discussed main events and drivers of CMA design and implementation. One author traced evolving formulation and interpretation of eligibility and approval criteria and coded which aspects were considered by parties as ambiguous. Codes and aspects were subsequently jointly discussed for interpretation.

Second, we analysed whether and how regulators (Section 4.3.1) and companies (Section 4.3.2) utilised CMA and acted upon ambiguities. Procedural and interview data from interviewees involved in procedures were used to reveal characteristics of assessment procedures resulting in CMA. We compared activities of regulators (e.g. voting,

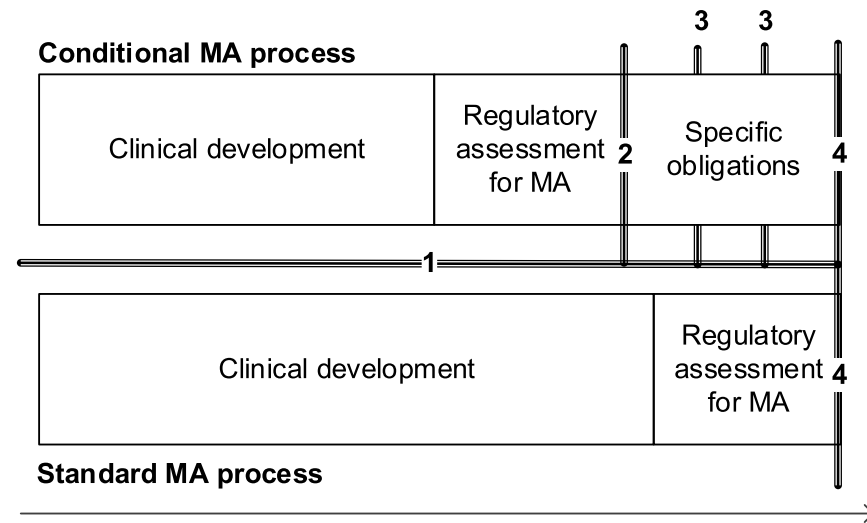

Comprehensiveness of data

Legend (numbers correspond to numbers in Figure):

1 Eligibility criteria for Conditional MA: Seriously debilitating or life-threatening diseases; Emergency situation; Orphan medicinal product

2-3 Approval criteria during initial Conditional MA (2) and annual renewal of Conditional MA (3): Benefit-risk balance is positive; Unmet medical needs will be fulfilled; Likely that applicant will provide comprehensive data; Benefits of immediate availability outweigh risks. License is valid for 1 year. Conversion to standard MA takes place when obligations are fulfilled and approval criteria for standard MA (4) are met.

4 Approval criteria for standard MA: Benefit-risk balance is positive. License is valid for 5 years

$\mathrm{MA}=$ marketing authorisation

Fig. 1. Schematic design of CMA.

review times) and companies (e.g. scientific advice, CMA requests) resulting in granting conditional versus standard MA. Both authors also read all interviews in full to contextualise the quantitative findings and jointly extracted reasons for why regulators and companies used CMA in particular ways.

Third, we examined regulatory outcomes and commonalities in utilisation patterns by assessing whether actions and outcomes were similar between procedures and depending on companies involved and products under assessment. We also examined archival documents pertaining to the evaluation phase of CMA (e.g. changing guidelines) to understand how utilisation patterns fed back on proposals and actions for further regulatory change (Section 4.5).

\section{Results}

\subsection{History of the law-making process}

Fig. 2 provides an overview of key events in the regulatory cycle. As Davis and Abraham (2013) have shown, the idea of creating CMA emerged from the '2001 pharma legislation review' initiated by the EC to review experiences with the first six years of the centralised procedure for EU-wide drug approval. One prominent issue put forward was that - unlike in the US - there was limited possibility in the EU to distinguish between drugs that were innovative in a pure technical sense and those deemed urgently needed because of a promise of therapeutic advance. Expedited approval was only allowed under 'exceptional circumstances', when additional data collection on patients was judged impossible for ethical or scientific reasons.

During the 2001 review process, widening the use of the exceptional circumstances procedure was proposed to increase possibilities for expedited approval. This was particularly considered necessary because of "legitimate expectations of patients" (EC, 2004). An impact assessment written by the EC also demonstrated a wish to use the instrument to 


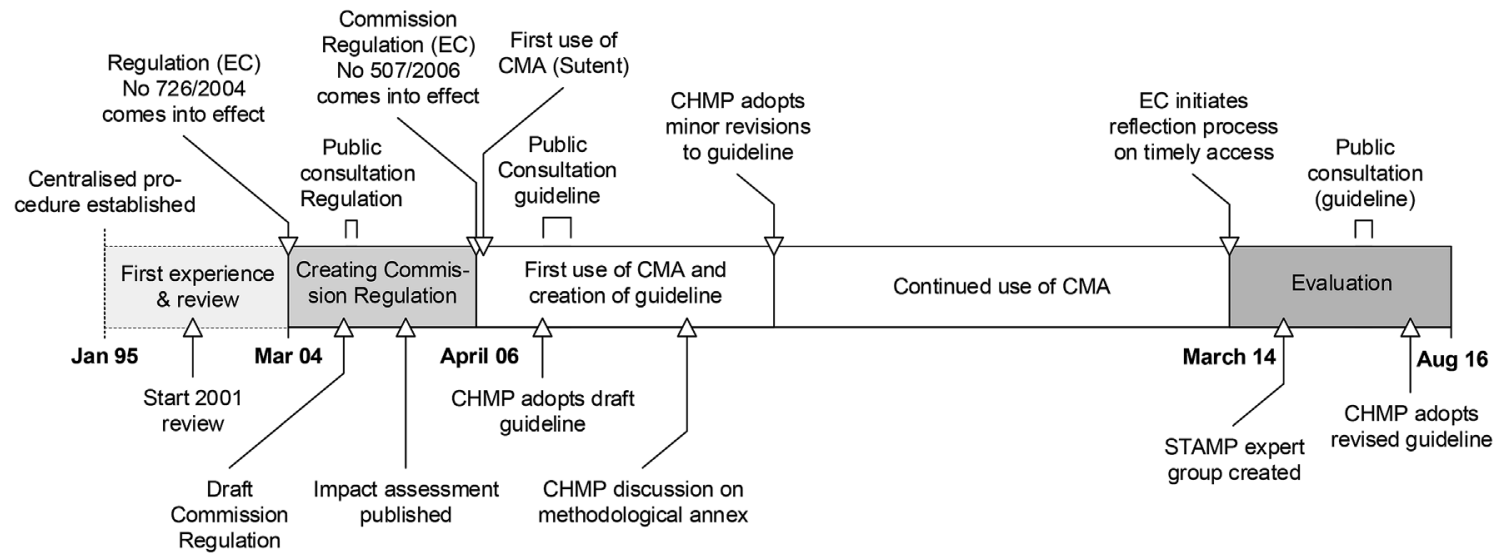

Fig. 2. Timeline of the CMA regulatory life-cycle.

counteract a stagnating number of drug approvals and declining competitiveness of the European pharmaceutical industry (EC, 2005). Moreover, harmonization of evidentiary standards in the context of the International Council of Harmonization of Technical Requirements for Registration of Pharmaceuticals for Human Use (ICH) played a role since this would allow companies to use the same evidence for obtaining approval in multiple jurisdictions (see Davis and Abraham, 2013 for a discussion on relative importance).

Interestingly, broad use of the exceptional circumstance procedure already occurred as indicated by a relatively high proportion of products $(14 \%)$ that were judged as 'exceptional' by regulators (Garattini and Bertele, 2001; Davis and Abraham, 2013). A survey signalled that regulators were reluctant to enshrine this informal practice in law because they feared that companies "would abuse follow-up commitments" (EC, 2000, p. 174) once drugs were authorised on the market. Fears followed from limited legal possibilities for drug withdrawal and enforcement of conducting post-marketing studies. They were fuelled by FDA's experience indicating that a substantial amount of post-marketing studies were not timely fulfilled or started at all (FDA, 2004).

To mitigate concerns, political debates converged on a proposal for a completely new form of MA that would be valid for one instead of five years and conditional on the fulfilment of specific obligations to be reviewed annually by EMA. This new MA form was adopted by the European Council and Parliament as part of Regulation No 726/2004 (European Parliament and Council, 2004). The Regulation did provide general provisions and noted that the MA form would be reserved to drugs of "major therapeutic interest" (EC, 2004). It gave mandate to the EC to further develop the MA form in a separate Commission Regulation. We elaborate next on how the EC proposals were received by stakeholders and how this process contributed to the formulation of ambiguous eligibility and approval criteria in the final Commission Regulation adopted in March 2006 (EC, 2006).

\subsection{Emergence of ambiguities}

When the EC presented a draft regulation in November 2004 it limited CMA eligibility to products for seriously debilitating or lifethreatening diseases, orphan medicinal products and products to be used in emergency situations. The draft also stipulated that applicants needed "to demonstrate the public health interest of the medicinal product" (EC, 2004), echoing Regulation No 726/2004. Approval criteria stated that the applicant should be "able to demonstrate a presumed positive benefit-risk balance based on scientific evidence and pending completion of further studies" (EC, 2004).

A public consultation on the draft made clear that the regulation was met with mixed responses. Particularly some public health advocates, organised consumer groups and reimbursement agencies expressed concerns that the definition of "public health interest" was not sufficiently specified, and eligibility for CMA too broad (EC, 2005). Public health advocates and reimbursement agencies feared that the use of the instrument for products treating seriously debilitating or lifethreatening diseases would unnecessarily increase risks for patients of inadequately assessed drugs and complicate reimbursement. They argued that CMA should be reserved to approval of orphan drugs for which it is inherently difficult to provide sufficient data within reasonable timeframes. Public health advocates also considered the draft regulation too unspecific and permissive concerning the proposed approval criterion that drugs could be authorised based on a "presumed positive benefit-risk balance". They requested more details on the evidence necessary for approval, e.g. whether randomised controlled trials (RCTs) based on clinical endpoints would still be necessary for CMA.

While industry organisations welcomed the proposal, they did express concerns about the concepts "public health interests" and "seriously debilitating or life-threatening diseases". They suggested to make CMA eligible for products that fulfil unmet medical needs and at the same time to use "demonstration of unmet medical need" as an approval criteria. This suggestion signalled a wish to broaden eligibility and streamline standards with US expedited pathways where "unmet medical need" was already used as a concept for more than a decade.

Expressed concerns about the proposed criteria were considered in the final Commission Regulation in two ways. First, although eligibility criteria did not change, "demonstration of unmet medical need" came to replace "demonstration of public health interest" as approval criterion. Akin to US expedited pathways, unmet need was defined as a "lack of a satisfactory treatment for a patient population or a major therapeutic advantage over existing treatments when alternatives are available" (EC, 2006). Second, approval criteria for evidence generation became stricter. More specifically, the criterion the "applicant is able to demonstrate a presumed positive benefit-risk balance" (EC, 2004) was changed into "the risk-benefit balance is positive as defined in Article 1(28a) of Directive 2001/83/EC" (EC, 2006). This change was most probably made because it was not legally possible to introduce a new benefit-risk concept in a Commission Regulation only.

The changes however led to ambiguity among regulators and companies about what the regulation would exactly afford. Regarding the unmet medical need definition, major therapeutic advantage over existing treatments was experienced as ambiguous as it was difficult to reconcile with approval based on lower evidentiary requirements. Therapeutic advance for the same indication as an approved drug is conventionally established based on RCT data that compare new and existing treatments during late-stage clinical development. It was unclear how such data could be obtained in early development, and how regulators could assess it in the absence of such data.

Regarding evidentiary requirements, the change from "presumed positive benefit-risk balance" to "benefit-risk balance" generated ambiguity as the regulation now stipulated the same approval criteria for 
conditional as for standard MA, but did not specify how evidentiary requirements could deviate from standard approval. Put differently, the regulation did not specify how much less comprehensive data would be acceptable at initial authorisation.

EMA and CHMP were given considerable discretionary autonomy to specify evidentiary requirements of less comprehensive data and to operationalise and assess unmet medical needs. The discretionary autonomy particularly pertained to the design of clinical efficacy trials, such as whether data from Phase III trials were still necessary for granting CMA, and whether randomisation, clinical endpoints and comparators were needed. Discretionary autonomy was granted although the regulation was perceived as being in need of elaboration. Among firms there was an expectation that EU and US standards for expedited approval would become more aligned, but it was unclear whether such alignment could be realised in practice within the boundary of the regulation.

\subsection{Acting upon ambiguous standards}

\subsubsection{Regulators}

The scope for discretionary action led to debate within the CHMP on how to implement the new MA form. This was observable from the process of creating a guideline on CMA application. While a draft was adopted for consultation in December 2006 and public consultation ended in March 2007, it took more than two years before the guideline was finally adopted by CHMP (EMA, 2009). The main point of discussion within the CHMP pertained to the design of clinical efficacy trials for granting CMA. The CHMP started to write a document describing methodological issues regarding data requirements and trial design with the goal of including this as an appendix to the guideline (EMA, 2008). However, while the document was internally discussed among CHMP members on various occasions, it was never published because CHMP members were divided on the issue and some of the proposals were considered to fall outside the boundaries of the law.

As differences in interpretations went unresolved, EMA-CHMP adopted the guideline in 2009 without the methodological appendix. The stipulated data requirements for establishing a positive benefit-risk balance in the guideline now more or less corresponded to data requirements for a standard MA. The guideline mentioned that "the demonstration of a positive benefit-risk balance should be based on (comprehensive) scientific evidence, in particular evidence from therapeutic confirmatory trials" (EMA, 2006, p. 3), corresponding to existing requirement for a standard MA. Regarding demonstration of unmet need, the guideline mentioned that "therapeutic advantages should be demonstrated over existing methods used in clinical practice (if any), using robust evidence, normally, from well conducted randomised controlled trials" (EMA, 2009, p. 5), seemingly contradicting the intention for approval based on less comprehensive data. Thus, EMA took a risk-averse approach in rule interpretation by not diverting too much from known standards and leaving ambiguities about the possibility for lower evidentiary requirements unresolved.

The observation that the CHMP was internally divided about the interpretation of standards is also visible from assessment procedures characteristics. Table 1 shows that between April 2006 and August 2016 recommendations for a standard MA were made by consensus by CHMP in $90 \%$ of procedures, while for CMA a consensus vote was only casted in $64 \%$ of procedures. Additionally, $16 \%$ of products were only recommended for CMA after CHMP reached an initial negative opinion and the company requested a data re-examination. Difficulties in reaching consensus among regulators is further illustrated by relatively long assessment procedures with a median [IOR] of 421 [329-491] days for CMA compared to 337 [281-400] days for standard MA. Such longer assessment procedures conflicted with the goal of CMA to provide expedited access. They demonstrate the difficulties and reluctance on the side of regulators to come to positive regulatory decisions on CMA, against the background of ambiguous standards. Regulators defended their stance by stressing that CMA was about abbreviated development rather than abbreviated assessment. Companies voiced expectations about conditional and accelerated assessment being combined more frequently stressing the need for accelerated access to drugs intended to address unmet medical needs.

Further illustration of how regulators acted upon ambiguous standards is provided by insights from procedures that resulted in CMA. On April 27, 2006 Sutent (sunitinib) was the first CMA recommended product by CHMP. Sutent was submitted by the company for two orphan designated indications: gastrointestinal stromal tumour (GIST) and metastatic renal cell carcinoma (MRCC). While clinical development for GIST was based on a RCT, the submitted results for MRCC were based on two phase II single-arm studies that showed an effect on overall response rate (ORR) as a surrogate endpoint. While the CHMP considered the data for GIST strong enough for approval, it consulted the Scientific Advisory Group Oncology to interpret the treatment effect for MRCC in the studied population. Based on this the CHMP concluded that the trials provided convincing evidence of benefit, but also that ORR could not be considered as a direct measure of clinical benefit. The CHMP therefore proposed the granting of a CMA as the benefit-risk balance was deemed positive for both indications, but comprehensive clinical data was not deemed available for the MRCC indication (EMA, 2007a).

The decision-making process of Sutent is illustrative of subsequent use of the CMA pathway in four ways. First, the CHMP proposed to grant a CMA for Sutent relatively late in the procedure. CMA was not proactively requested by the company, but recommended by CHMP as an outcome of the assessment procedure. This 'reactive' use of CMA by CHMP when submitted data are not strong enough to justify a standard MA became common practice. Table 1 shows that over the study period 16 out of 29 (55\%) products were recommended for CMA by CHMP without applicant's request. Second, in the absence of a CMA request, the demonstration of unmet medical need of drugs took place during and often at the end of the assessment procedure. Unmet medical need was not a criteria that drove the CMA, but rather an argument that was brought forward during the procedure to justify approval based on less comprehensive data. Thus, CMA seemed to emerge as a compromise between a standard approval and a refusal. Third, the decision to grant a CMA was only made after consulting a scientific advisory group which became a more general pattern in order to mitigate risks and reputational consequences associated with CMA granting. Fourth, at time of application a phase III RCT with Sutent in the MRCC indication was already ongoing. The ongoing study and expectation of results in due course reduced uncertainty among CHMP to grant approval based on less comprehensive data. Thus, an assessment of whether applicants were in a position to provide comprehensive data - which is one of the approval criteria for CMA (Fig. 1) - became important to grant CMA.

Asking for additional data from ongoing studies, interim results and post-hoc analyses from trials became a prominent way for CHMP members to deal with ambiguities and uncertainties associated with expedited approval. This is an observation which has also been described for the re-approval of Lotronex by the FDA (Davis and Abraham, 2013). In the case of CMA it is particularly visible in assessment procedures for non-orphan products that treat 'seriously debilitating and life-threatening diseases', the category that was already considered most ambiguous in the regulation-making process. In several CMA procedures applicants were asked to release top-line results on clinical efficacy available from ongoing Phase III trials, including early data on clinical endpoints such as overall survival. Promising data from these interim analyses subsequently provided the CHMP with confidence to recommend CMA. In other cases, the CHMP requested to stratify clinical trial data by patients' disease mutation in order to understand whether efficacy profiles were different for subpopulations. In case CMA was recommended based on such 'personalised' data, the indication was restricted to specific sub-populations in order to manage risks and define a population with unmet medical need. For instance, during 
Table 1

Comparison of assessment procedure by CHMP for medicines granted standard or conditional MA.

\begin{tabular}{|c|c|c|c|}
\hline & Standard $(n=265)$ & Conditional $(n=29)$ & p-value \\
\hline Median [IQR] length of CHMP assessment procedure (in days) & $337[281-400]$ & $421[329-491]$ & 0.014 \\
\hline Authorised with CHMP consensus* & $231(90 \%)$ & $18(64 \%)$ & $<0.001$ \\
\hline \multirow[t]{2}{*}{ Authorised based on first CHMP opinion } & $263(99 \%)$ & $24(83 \%)$ & $<0.001$ \\
\hline & Standard $(n=265)$ & Conditional $(n=29)$ & Not approved $(\mathrm{n}=82)$ \\
\hline Request for standard MA & $263(99 \%)$ & $16(55 \%)$ & $73(87 \%)$ \\
\hline Request for conditional MA & $2(1 \%)$ & $13(45 \%)$ & $9(13 \%)$ \\
\hline
\end{tabular}

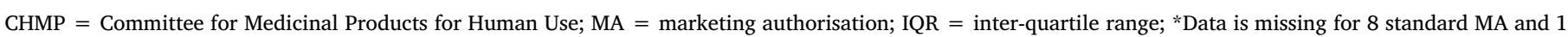
conditional MA.

a re-examination procedure the indication for Vectibix (panitumumab) was restricted to metastatic colorectal carcinoma with non-mutated (wild-type) KRAS after post-hoc analyses were performed (EMA, 2007b), while the indication for Caprelsa (vandetanib) includes a statement that a potential lower benefit might be observed for patients in whom Rearranged during Transfection (RET) mutation is not known or is negative (EMA, 2012).

Thus, what emerges from these cases is that there was considerable ambiguity among regulators over the interpretation of approval criteria for CMA. These ambiguities were not resolved in a guidance document as the CHMP was internally divided, and interpretations of regulators and their political principals were not aligned. Consequently, regulators started to utilise CMA in unforeseen ways and in the majority of cases as an alternative approval option for drugs that they deemed unsuitable for standard MA. In doing so, they used the instrument as a 'consolation prize' or 'rescue option' for drugs when data was not strong enough to recommend standard approval, in the process forcing companies to generate further evidence on the clinical effects of drugs.

\subsubsection{Companies}

In the majority of approvals, companies did not prospectively apply for CMA (Table 1). Rather, they adopted a 'wait-and-see' approach, not signalling upfront that their clinical data was less comprehensive. This was surprising given that industry associations and companies actively lobbied for CMA in the law-making phase and endorsed its pro-active use in the consultation.

The analyses reveals that in light of ambiguity about eligibility and approval standards, companies often considered a prospective CMA application too risky and strategically refrained from applying. A dedicated development program to obtain CMA based on less comprehensive data was perceived as increasing the risk of non-approval by CHMP, of negative decisions by national reimbursement agencies, and difficult to reconcile with development strategies for the US market: "There is not so much point in getting conditional [..] if other stakeholders [..] say we want the standard [..]. There needs to be that broader understanding and acceptance" (interview results). The strategy of not requesting CMA was also sometimes opportunistic as applicants preferred obtaining a standard MA with a validity of five years and less strict post-marketing follow-up over a CMA: "If you request upfront you [..] only [...] get conditional, aren't you? [..] commercially you are giving yourself broader options" (interview results). Companies therefore chose not to signal that their dossier contained less comprehensive data. Dossiers were also sometimes intentionally submitted prematurely because of a race with competitors to be a firstmover on the market, because further development of the drug was not deemed valuable enough or because endorsement by regulators was necessary to generate milestone payments and/or funding.

Table 2 shows that a 'wait-and-see' approach was particularly observed among experienced companies who developed non-orphan drugs for seriously debilitating or life-threatening diseases as compared to less experienced companies developing orphan drugs. Less experienced companies were more likely to engage in early dialogue with regulators about their clinical development program and to proactively request CMA. Paradoxically, these companies were less likely to obtain drug approval. Table 2 demonstrates that companies requesting CMA had on median less approval experience compared to companies requesting standard MA. In contrast, companies receiving CMA had on median more experience compared to those receiving standard MA, and to those obtaining no approval. Thus, experienced companies were better able to obtain CMA for their products despite the fact that they showed less interest in the instrument.

So, experienced companies seemed to have a different approach to dealing with ambiguity than less experienced companies. Our findings suggest a number of potential facilitating factors. First, large companies experienced more possibilities to behave opportunistically because they could more easily absorb the (financial) consequences of delayed approval. Second, in case CMA was used as a 'rescue option', regulators often asked for additional evidence. Large companies were better able to respond in a timely manner to such requests given readily available data, resources and capabilities. Third, when discussing about specific obligations to be fulfilled in the post-marketing phase, regulators tended to put confidence in companies who had Phase III trials already ongoing, or at least planned, as formalised in the approval criteria which stated that applicants should be in a position to provide comprehensive data. Thus, the use of this approval criteria facilitated that advantages for incumbent parties became institutionalised in the decision-making process.

\subsection{Regulatory outcomes and directions of change}

The presented analysis shows how discretionary actions by regulators and companies following from ambiguous standards resulted in the emergence of two different routes to expedite approval within a single pathway and instrument. Table 3 summarises some stylised differences between the two routes to CMA.

The prospective approval route is a form of CMA by design which emerges from open communication between regulators and companies from early stages of clinical development onwards. Addressing unmet medical needs is the driver of clinical development and ambiguity about eligibility for CMA is limited. An outcome of prospective use is that regulators seem to be willing to accept considerable risks and uncertainties and to shift the generation of relatively large pieces of evidence such as new phase III trials to the post-marketing phase. The model is particularly used by less experienced companies, often developing orphan medicinal products. In the study period it is associated with relatively low approval rates as the CHMP often did not consider the submitted data strong enough for obtaining CMA and companies experienced difficulties in submitting additional pieces of evidence during the procedure.

The reactive approval route is more often used by experienced companies who develop non-orphan drugs for seriously debilitating and life-threatening diseases. Communication between regulators and 
Table 2

Comparison of applications with request for conditional and standard MA.

\begin{tabular}{|c|c|c|c|c|c|c|c|}
\hline & \multicolumn{3}{|c|}{ Request by applicant } & \multicolumn{4}{|c|}{ CHMP decision } \\
\hline & $\begin{array}{l}\text { Standard } \\
(\mathrm{n}=352)\end{array}$ & $\begin{array}{l}\text { Conditional } \\
\text { MA }(n=24)\end{array}$ & p-value & $\begin{array}{l}\text { Standard } \\
(n=265)\end{array}$ & $\begin{array}{l}\text { Conditional } \\
\quad(n=29)\end{array}$ & $\begin{array}{l}\text { Not approved } \\
\quad(n=82)\end{array}$ & p-value \\
\hline Scientific advice or protocol assistance & $235(67 \%)$ & $22(92 \%)$ & 0.011 & $186(70 \%)$ & $20(69 \%)$ & $51(62 \%)$ & 0.396 \\
\hline Orphan drug designation & $118(34 \%)$ & $18(75 \%)$ & $<0.001$ & $84(32 \%)$ & $17(59 \%)$ & $35(43 \%)$ & 0.006 \\
\hline $\begin{array}{l}\text { Median }[\mathrm{IQR}] \text { number of earlier successful MAs at } \\
\text { EMA* }\end{array}$ & $4[0-13]$ & $1[0-10.5]$ & 0.334 & $5[0-14]$ & $8[1-17]$ & $0[0-4]$ & $<0.001$ \\
\hline
\end{tabular}

$\mathrm{MA}=$ marketing authorisation; * Total number of successful MAs at EMA (since 01-01-1995) of the applicant at time of application

Table 3

Two stylised routes to CMA.

\begin{tabular}{|c|c|c|}
\hline & Prospective & Reactive \\
\hline Dialogue & Open/Early & Strategic/Absent \\
\hline Eligibility & Certain & Contested \\
\hline Unmet needs & Driver of procedure & Outcome of procedure \\
\hline Evidence & Phase II data & Early data from Phase III \\
\hline Approval & Limited approval rates & 'Precision' access $\mathrm{N}^{\text {th }}$ line access \\
\hline Obligations & $\begin{array}{l}\text { Relatively large studies } \\
\text { New studies }\end{array}$ & $\begin{array}{l}\text { Ongoing studies Clinical } \\
\text { development portfolio }\end{array}$ \\
\hline Firm experience & Less experienced & More experienced \\
\hline Products & $\begin{array}{l}\text { Orphan medicinal } \\
\text { products }\end{array}$ & $\begin{array}{l}\text { Non-orphan seriously debilitating } \\
\text { and life-threatening diseases }\end{array}$ \\
\hline Importance & Increasing over time & Decreasing over time \\
\hline
\end{tabular}

companies during clinical development is less pronounced, more strategic or even absent. It is less clear whether products submitted for MA fall within the scope of a CMA and companies generally do not signal this to regulators. Consequently, unmet medical need according to CMA criteria is not a driver of CMA, but determined after regulators conclude that data is not comprehensive enough to grant a standard MA. Regulators tend to be internally divided about drug approval and sometimes find compromise by narrowing down indications to an $\mathrm{N}^{\text {th }}$ line treatment or a genetically defined sub-population based on additional data provided during the procedure. Regulators also try to limit the risk that data from post-marketing studies are not generated or not produced within agreed upon timeframes by favouring approval of drugs for which Phase III trials are already running.

The fact that in the majority of cases CMA was used as a 'rescue option' was taken up when the EC called into being an expert group for a comprehensive evaluation of instruments for "Safe and Timely Access to Medicines for Patients" (STAMP) in 2014. During the first meeting it was noted that the perception of CMA among firms and other stakeholders was negative pertaining amongst others to the fact that CMA was not used pro-actively. It was concluded that "prospective planning of CMA, scientific advice and early dialogue could improve the use of CMA" (EC, 2015). This was subsequently formalised in the issuance of a revised guideline on the application of CMA in which applicants are encouraged to prospective plan CMA in order to "avoid delays in assessment procedures" (EMA, 2016, p. 10). The change did however not make prospective planning a requirement for CMA and while reactive use of CMA seems to be decreasing over time it did not halt after the guideline revision.

At the same time, the revised guideline also clarified the interpretation of eligibility and approval criteria for CMA within the boundaries of the legal framework. In the revised guideline issued by EMA-CHMP a possibility for approval based on intermediate endpoints is included which "are reasonably likely to translate into clinical benefit, but do not directly measure the clinical benefit" (EMA, 2016, p. 6). This harmonises criteria for expedited approval in the EU and US. Eligibility criteria for CMA were also extended to a second or subsequent drug addressing the same unmet medical need as long as the first-in-class product is still conditional, while "major improvements to patient care" was added to the definition of therapeutic advantage. Regarding unmet medical need it was noted that whether such needs are actually addressed is only "gradually confirmed after authorisation" (EMA, 2016, p. 9). This suggests that resolving of ambiguities coincided with a broadening of the interpretation of the scope of CMA within the existing legal framework.

\section{Conclusion}

This longitudinal analysis of the implementation of CMA in the EU showed how ambiguous legal standards for expedited drug approval provided scope for interest-driven discretionary action by regulators and companies, resulting in the emergence of two different routes to grant expedited approval within a single instrument. The chain of events that we document reveals that the introduction of more flexible and de-regulatory options for drug development and regulation by the EC were initially not met with enthusiasm by regulators and companies due to normative and interpretative ambiguities about eligibility and approval criteria. These ambiguities triggered avoidance and opportunistic strategies among companies who in the majority of cases did not signal to regulators when their products were developed based on less comprehensive data. Pressured by their political principals, regulators however actively worked to utilise CMA by employing it as a rescue option in lengthy and uncertain approval procedures when submitted evidence was not strong enough to grant standard approval. They took on enabling roles and corresponding responsibilities in those procedures and, by doing so, facilitated approval of personalised medicines and niche products in procedures characterised by fluid role divisions with regard to how to reduce uncertainties about the clinical effects of medicines.

These findings enhance our understanding of how ambiguity of drug approval standards evolve during implementation of a new regulatory instrument in three ways. First, regarding the temporal versus permanent nature of ambiguity, we showed that when there is limited buy-in of parties ambiguities can remain unresolved for a considerable period of time. In the case of CMA, ambiguity provided room for unexpected and under-utilization of the instrument as well as the emergence of a negative perception of the instrument. The negative experience was subsequently used to argue for less strict interpretations of the regulation, following directions already envisioned, yet not realised, during the discussions on the draft regulation. Second, one reason for the more permanent character of ambiguity in our case seems to be the absence of commitments between regulators and companies and learning conditions. More specifically, our study suggests that the combination of voluntary and non-binding dialogues between companies and regulators and degrees of freedom to choose between approval pathways increased the possibility for strategic commitments and opportunistic behaviour. This observation has previously been made in the context of the co-existence of and overlap between the three drug approval procedures at national and EU-wide level (Abraham and Lewis, 2000). Third, we showed that assessing drugs based on 'non-traditional' forms 
of regulatory knowledge (Hauray, 2017), such as considerations of patient needs and capabilities of companies to conduct studies increased the level of ambiguity among involved parties. With increasing uncertainties about the benefit-risk ratio of drugs in expedited pathways, these 'non-traditional' considerations have become more important. In our study, this did however not result in robust operationalization of these criteria and their use as starting point to consider CMA. Rather these criteria seem to be used in a more ad-hoc fashion as a means to legitimise approval. Furthermore, their use also seemed to result in advantages for larger companies as they were better able to deal with regulators requests for additional data and studies.

It is currently unclear how the described CMA utilization patterns affected the uptake of CMA approved medicines and their public health impact in the EU. Regarding safety, to date none of the drugs approved through CMA have witnessed a safety-related withdrawal. A study by Boon et al. (2010) also showed that the use of CMA is not associated with a higher likelihood of detecting unexpected serious safety issues after approval. Regarding efficacy, a recent study by Davis et al. (2017) showed that for none of 10 CMA approved oncology drugs, clinical evidence was available that showed improvement in quantity or quality of life after a minimum of 3 years of approval. Thus, although we do not claim in this paper that CMA approval of any particular drug was not justified, uncertainties about the therapeutic value of these drugs can remain unresolved after approval. Reactive use of CMA likely contributes to this as it inhibits systematic and consistent assessment of how unmet medical needs are actually addressed upon drug approval. Such use also demonstrates how interest-driven actors can act upon ambiguities in attempts to shape regulatory outcomes and stretch rule interpretations.

\section{Acknowledgements}

All authors have completed the ICMJE uniform disclosure form at www.icmje.org/coi_disclosure.pdf and declare: $\mathrm{JH}$ is financed by a VENI grant (451-15-037) from the Netherlands Organisation for Scientific Research (NWO). Interviews on the functioning of the conditional marketing authorisation have been conducted within the context of two previous studies financed by the Netherlands Organisation for Scientific Research (NWO) responsible Innovation program and "Escher Projects" which is part of the Dutch public-private partnership Lygature and has received funding from European Federation of Pharmaceutical Industry \& Associations (EFPIA) and the Association of the European Self-Medication Industry (AESGP). There are no other financial relationships with any organisations that might have an interest in the submitted work in the previous three years and no other relationships or activities that could appear to have influenced the submitted work.

\section{References}

Abraham, J., 1995. Science, Politics and the Pharmaceutical Industry: Controversy and Bias in Drug Regulation. UCL Press.

Abraham, J., 2008. Sociology of pharmaceuticals development and regulation: a realist empirical research programme. Sociol. Health Illness 30, 869-885.

Abraham, J., Lewis, G., 2000. Regulating Medicines in Europe: Competition, Expertise and Public Health. Routledge.

Baird, L.G., Banken, R., Eichler, H.-G., Kristensen, F.B., et al., 2014. Accelerated access to innovative medicines for patients in need. Clin. Pharmacol. Ther. 96, 559-571.

Boon, W.P.C., Moors, E.H.M., Meijer, A., Schellekens, H., 2010. Conditional approval and approval under exceptional circumstances as regulatory instruments for stimulating responsible drug innovation in Europe. Clin. Pharmacol. Ther. 88, 848-853.

Braillon, A., Menkes, D.B., 2016. Balancing accelerated approval for drugs with accelerated withdrawal. JAMA Intern. Med. 176, 566-567.

Carpenter, D.P., 2004a. The political economy of FDA drug review: processing, politics, and lessons for policy. Health Aff. (Millwood) 23, 52-63.

Carpenter, D.P., 2004b. Protection without capture: product approval by a politically responsive, learning regulator. Am. Pol. Sci. Rev. 98, 613-631.

Chiti, E., 2013. European agencies' rulemaking: powers, procedures and assessment. Eur. Law J. 19, 93-110.

Daemmrich, A.A., 2004. Pharmacopolitics: Drug Regulation in the United States and
Germany. Univ of North Carolina Press.

Davis, C., Abraham, J., 2013. Unhealthy Pharmaceutical Regulation: Innovation, Politics and Promissory Science. Palgrave Macmillan.

Davis, C., Naci, H., Gurpinar, E., Poplavska, E., Pinto, A., Aggarwal, A., 2017. Availability of evidence of benefits on overall survival and quality of life of cancer drugs approved by European Medicines Agency: retrospective cohort study of drug approvals 200913. BMJ 359, j4530.

de Vrueh, R., Baekelandt, E.R.F., de Haan, J.M.H., 2013. Background Paper 6.19 Rare Diseases. WHO Priority Medicines for Europe and the World.

EC, 2000. Evaluation of the operation of Community procedures for the authorization of medicinal products. In: On Behalf of the EC by CMS Cameron McKenna and Andersen Consulting. EC, DG Enterprise, Brussels October 2000.

EC, 2004. Draft Commission Regulation (EC) No 507/2006 on the Conditional Marketing Authorisation Regulation for Medicinal Products for Human Use Falling within the Scope of Regulation (EC) No 726/2004 of the European Parliament and of the Council.

EC, 2005. Impact Assessment on a Proposal for a Commission Regulation on the CMA for Medicinal Products Falling within the Scope of Regulation. (EC) No 726/2004.

EC, 2006. Commission regulation (EC) No 507/2006 of 29 March 2006 on the CMA for medicinal products for human use falling within the scope of regulation (EC) No 726/ 2004 of the european parliament and of the Council. Off. J. L/92, 6-9.

EC, 2015. STAMP Commission Expert Group Summary Record. European Commission DG Health and Food Safety.

Ehmann, F., Papaluca Amati, M., Salmonson, T., Posch, et al., 2013. Gatekeepers and enablers: how drug regulators respond to a challenging and changing environment by moving toward a proactive attitude. Clin. Pharmacol. Ther. 93, 425-432.

Eichler, H.-G., Pignatti, F., Leufkens, H., Breckenridge, A., 2008. Balancing early market access to new drugs with the need for benefit/risk data: a mounting dilemma. Nat. Rev. Drug Discov. 7, 818-826.

Eichler, H.-G., Oye, K., Baird, L.G., Abadie, E., et al., 2012. Adaptive licensing: taking the next step in the evolution of drug approval. Clin. Pharmacol. Ther. 91, 426-437.

EMA, 2006. Draft Guideline on the Scientific Application and the Practical Arrangements Necessary to Implement Commission Regulation (EC) No. 507/2006. Doc. Ref. EMEA/509951/2006.

EMA, 2007a. Sutent - EPAR: Scientific Discussion.

EMA, 2007b. Vectibix - EPAR: Scientific Discussion.

EMA, 2008. CHMP June 2008 Plenary Meeting Monthly Report. EMEA/CHMP/327265/ 2008.

EMA, 2009. CHMP May 2009 Plenary Meeting Monthly Report. EMEA/CHMP/330510/ 2009.

EMA, 2012. Caprelsa - EPAR: Scientific Discussion.

EMA, 2016. Guideline on the Scientific Application and the Practical Arrangements Necessary to Implement Commission Regulation (EC) No 507/2006. EMA/CHMP/ 509951/2006, Rev.1.

European Parliament and Council, 2004. Regulation (EC) No 726/2004 of the European Parliament and of the Council of 31 March 2004 laying down Community procedures for the authorisation and supervision of medicinal products for human and veterinary use and establishing a European Medicines Agency. Off. J. L 136, 1-33.

FDA, 2004. Report on the performance of drug and biologics firms in conducting postmarketing commitment studies. US FDA. Fed. Regist. 69 (50).

Garattini, S., Bertele, V., 2001. Adjusting Europe's drug regulation to public health needs. Lancet 358, 64-67.

GAO, Report, 2015. Drug Safety- FDA Expedites Many Applications, but Data for PostApproval Oversight Need Improvement. GAO-16-192.

Grin, J., Loeber, A., 2006. Theories of policy learning: agency, structure and change. In: Handbook of Public Policy Analysis: Theory, Politics, and Methods. CRC Press, pp. 201-219.

Hamburg, M.A., Sharfstein, J.M., 2009. The FDA as a public health agency. N. Engl. J. Med. 360, 2493-2495.

Hauray, B., 2017. From regulatory knowledge to regulatory decisions: the European evaluation of medicines. Minerva 55 (2), 187-208.

Hoekman, J., Boon, W., Bouvy, J., Ebbers, H., de Jong, J., De Bruin, M., 2015. Use of the conditional marketing authorization pathway for oncology medicines in Europe. Clin. Pharmacol. Ther. 98, 534-541.

Hoekman, J., Klamer, T.T., Mantel-Teeuwisse, A.K., Leufkens, H.G.M., De Bruin, M.L., 2016. Characteristics and follow-up of postmarketing studies of conditionally authorized medicines in the EU. Br. J. Clin. Pharmacol. 82, 213-226.

Irwin, A., Rothstein, H., Yearley, S., McCarthy, E., 1997. Regulatory science-towards a sociological framework. Futures 29, 17-31.

Kesselheim, A.S., Wang, B., Franklin, J.M., Darrow, J.J., 2015. Trends in utilization of FDA expedited drug development and approval programs, 1987-2014: cohort study. BMJ 351, h4633.

Kondo, H., Hata, T., Ito, K., Koike, H., Kono, N., 2017. The current status of sakigake designation in Japan, PRIME in the European Union, and breakthrough therapy designation in the United States. Ther. Innov. Regul. Sci. 51, 51-54.

Liberti, L., 2017. Globally Applicable Facilitated Regulatory Pathways to Improve Equitable Access to Medicines. Utrecht University, Utrecht.

Mahoney, J., Thelen, K., 2010. Explaining Institutional Change: Ambiguity, Agency, and Power. Cambridge University Press.

Mostaghim, S.R., Gagne, J.J., Kesselheim, A.S., 2017. Safety related label changes for new drugs after approval in the US through expedited regulatory pathways: retrospective cohort study. BMJ 358, j3837.

Naci, H., Wouters, O.J., Gupta, R., Ioannidis, J.P. a., 2017. Timing and characteristics of cumulative evidence available on novel therapeutic agents receiving Food and drug administration accelerated approval. Milbank Q. 95, 261-290.

Ossege, C., 2015. Driven by expertise and insulation? The autonomy of European Regulatory Agencies. Polit. Gov. 3, 101.

Stigler, G.J., 1971. The theory of economic regulation. Bell J. Econ. 2 (1), 3-21. 